\title{
Localization induced by magnetic fields in carbon nanotubes
}

\author{
Magdalena Margańska, Miriam del Valle, Sung Ho Jhang, Christoph Strunk, and Milena Grifoni \\ University of Regensburg, D-93053 Regensburg, Germany
}

(Received 4 March 2011; revised manuscript received 21 March 2011; published 23 May 2011)

\begin{abstract}
The electronic spectra of long carbon nanotubes (CNTs) can, to a very good approximation, be obtained using the dispersion relation of graphene with both angular and axial periodic boundary conditions. In short CNTs one must account for the presence of open ends, which may give rise to states localized at the edges. When a magnetic field is applied parallel to the tube axis, it modifies both momentum quantization conditions, causing hitherto extended states to localize near the ends. We study analytically and numerically the appearance and evolution of this peculiar localization phenomenon in CNTs of any nonarmchair chirality, including the electron spin. Conductance calculations show different evolution of spin up and down states in increasing magnetic field.
\end{abstract}

DOI: 10.1103/PhysRevB.83.193407

PACS number(s): 73.63.Fg, 73.23.Ad, 75.47.-m, 85.75.-d

The existence of geometry-induced localized states at the zigzag edge of graphene nanoribbons has been predicted some years ago, 1,2 recently seen experimentally and shown to influence the transport in graphene quantum dots. ${ }^{3}$ Similar states at the ends of single-wall nanotubes have been observed ${ }^{4}$ and studied. ${ }^{5-8}$ In zigzag-armchair nanotube junctions, the interface states calculated to appear at the junction were identified with the end states of the zigzag nanotube fragment. ${ }^{9,10}$

In this work we study another type of edge states, namely those which arise when initially extended states become localized in a parallel magnetic field. ${ }^{11,12} \mathrm{We}$ present a detailed analytical and numerical study of this effect in CNTs of arbitrary nonarmchair chirality, including the spin-orbit coupling and the Zeeman effect. We find that this phenomenon occurs also in those chiral CNTs which have no localized end states when the magnetic field is absent. Numerical calculations of the conductance of finite CNT devices in a continuously varying magnetic field suggest the possibility of spin-selective transport.

The model. Our starting point is the tight-binding Hamiltonian for a honeycomb lattice with one $p_{z}$ orbital per atom and with the interatomic potential $\hat{V}$. If we calibrate our energy scale so that the on-site energies vanish, the Hamiltonian is given by

$$
\hat{H}=\sum_{i \neq j} t_{i j}\left|z_{j}\right\rangle\left\langle z_{i}\right|
$$

where $i$ and $j$ are the lattice site indices, $\left|z_{j}\right\rangle$ is a $p_{z}$ orbital at site $j$, and $t_{i j}=\left\langle z_{j}|\hat{V}| z_{i}\right\rangle$ is the hopping integral between the sites. This Hamiltonian nicely captures the properties of flat graphene and CNTs. In order to properly describe finite size nanotubes in the magnetic field it is necessary to include the Peierls phase and curvature effects in the hopping elements $t_{i j}$. We follow here the approach of Ando. ${ }^{13}$ For the sake of clarity we shall initially neglect the spin-orbit coupling and the Zeeman effect, as they do not change our main conclusion. The spin-dependent effects will be addressed later.

The graphene coordinate system and the relevant real space vectors are shown in Fig. 1(a), while the graphene Brillouin zone with $K$ and $K^{\prime}$ points is shown in Fig. 1(b). In order to find the appropriate boundary conditions and eigenstates of CNTs we use an approach based on the Dirac equation treatment. ${ }^{2,14}$
Parallel magnetic field. The magnetic field modifies all hopping integrals by a Peierls phase factor. Its form can be derived using the substitution $\mathbf{p} \rightarrow \mathbf{p}-e \mathbf{A}$ and reads

$$
t_{i j}(\mathbf{B})=t_{i j}(0) \exp \left\{\frac{i e}{\hbar} \int_{\mathbf{r}_{i}}^{\mathbf{r}_{j}} \mathbf{A}(\mathbf{r}) \cdot d \mathbf{r}\right\} .
$$

In the cylindrical coordinates $(r, \varphi, z)$, with the $z$ direction aligned with the axis of the nanotube, a parallel magnetic field has coordinates $\mathbf{B}=(0,0, B)$. In the tangential gauge this gives $\mathbf{A}=(0, B r / 2,0)$. The Peierls phase then becomes

$$
\frac{i e}{\hbar} \int_{\mathbf{r}_{i}}^{\mathbf{r}_{j}} \mathbf{A}(\mathbf{r}) \cdot d \mathbf{r}=i \frac{\phi}{\phi_{0}}\left(\varphi_{j}-\varphi_{i}\right),
$$

where $\phi$ is the magnetic flux threading the nanotube, $\phi_{0}=h / e$ the flux quantum, and $\varphi_{j}-\varphi_{i}$ is the difference between the angular coordinates of site $j$ and site $i$.

Curvature. In a nanotube the $\sigma$ bonds are not orthogonal to the $p_{z}$ orbitals and the hopping integral $t_{i j}$ can be expressed as

$$
t_{i j}(0)=\left\langle z_{j}|\hat{V}| z_{i}\right\rangle=V_{\pi} \mathbf{n}_{i \mathrm{n}} \cdot \mathbf{n}_{j \mathrm{n}}+V_{\sigma} \mathbf{n}_{i \mathrm{t}} \cdot \mathbf{n}_{j \mathrm{t}},
$$

where $V_{\pi}$ and $V_{\sigma}$ are hopping parameters for the corresponding bonds. ${ }^{13}$ In our calculations we shall use the parameters from Ref. $15, V_{\sigma}=6.38 \mathrm{eV}$ and $V_{\pi}=-2.66 \mathrm{eV}$. The vector $\mathbf{n}_{i}$ is a unit vector normal to the nanotube surface at the site $i$. The components $\mathbf{n}_{i n}$ (normal) and $\mathbf{n}_{i t}$ (tangential) are defined with respect to a plane containing the $\sigma$ bond between $i$ and $j$ and parallel to the CNT axis. The hopping integral $t_{i j}(0)$ then reads

$$
\begin{aligned}
\left\langle z_{j}|\hat{V}| z_{i}\right\rangle= & V_{\pi} \cos \left(\varphi_{i}-\varphi_{j}\right) \\
& -\left(V_{\sigma}-V_{\pi}\right) \frac{R^{2}}{a_{c}^{2}}\left[1-\cos \left(\varphi_{i}-\varphi_{j}\right)\right]^{2},
\end{aligned}
$$

where $R$ is the nanotube radius and $a_{c}=1.42 \AA$ is the bond length in graphene.

In order to find the CNT spectrum it is convenient to express the Hamiltonian in the Bloch wave basis. The Bloch waves for the CNT sublattice $p$ are given by ${ }^{16}$

$$
\left|\Phi_{p}(\mathbf{k})\right\rangle=\frac{1}{\sqrt{N}} \sum_{i=1}^{N} e^{i \mathbf{k} \cdot \mathbf{r}_{p i}}\left|z_{p i}\right\rangle,
$$



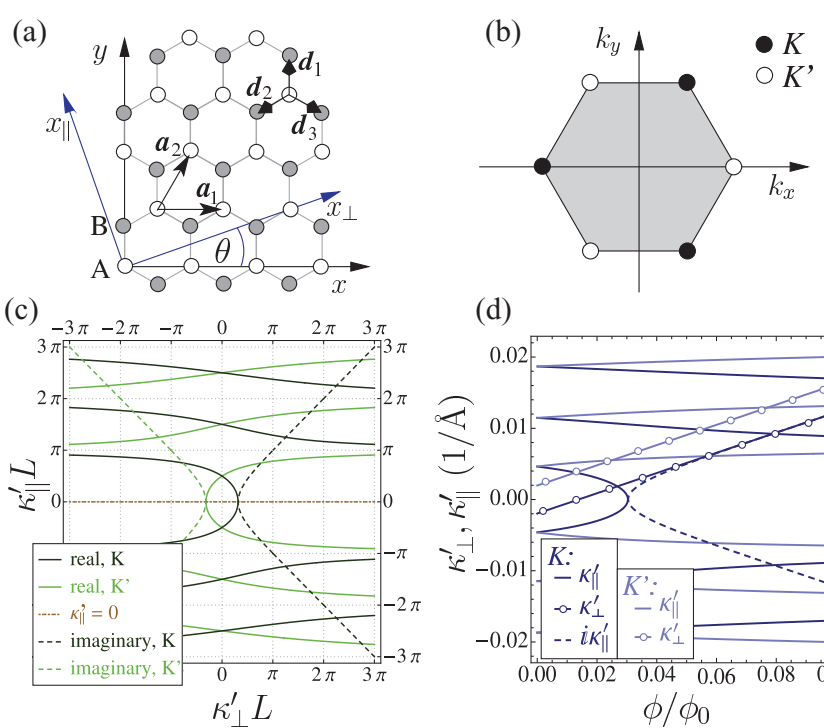

(d)

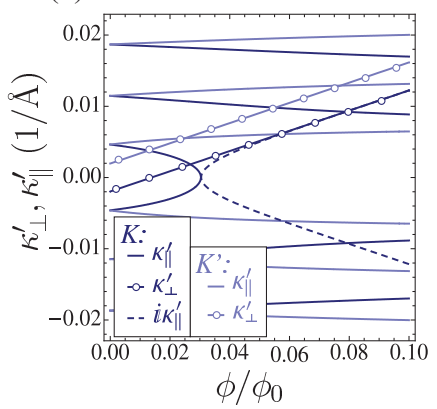

FIG. 1. (Color online) (a) Fragment of a graphene lattice. When considering a CNT with chiral angle $\theta$, we shall use the system of coordinates defined by directions perpendicular $\left(x_{\perp}\right)$ and parallel $\left(x_{\|}\right)$ to the tube axis. (b) Brillouin zone of graphene. (c) Real and imaginary solutions of Eq. (17), determining the quantization of $\kappa_{\|}^{\prime}$ as a function of $\kappa_{\perp}^{\prime}$. (d) Some of the real and imaginary solutions of Eq. (17) for the Fermi subband $\left(\kappa_{\perp}=0\right)$ of an $(18,0)$ CNT with 100 unit cells, with $\kappa_{\perp}^{\prime}$ and $\kappa_{\|}^{\prime}$ as functions of the magnetic flux $\phi$.

where $N$ is the number of the unit cells. The Bloch wave on the whole lattice is a linear combination of Bloch waves on individual sublattices and can be written as

$$
|\Phi(\mathbf{k})\rangle=\sum_{p=A, B} \eta_{p}(\mathbf{k})\left|\Phi_{p}(\mathbf{k})\right\rangle=\left(\begin{array}{c}
\eta_{A}(\mathbf{k}) \\
\eta_{B}(\mathbf{k})
\end{array}\right) .
$$

In this basis the Hamiltonian acquires the form,

$$
\hat{H}(\mathbf{k})=\left(\begin{array}{cc}
0 & H_{A B}(\mathbf{k}) \\
H_{A B}^{\dagger}(\mathbf{k}) & 0
\end{array}\right)
$$

where $H_{A B}(\mathbf{k})=\sum_{i=1}^{3} t_{i} e^{i \mathbf{k} \cdot \mathbf{d}_{i}}, \mathbf{d}_{i}$ are the vectors connecting an $A$ sublattice atom with its neighbors, as shown in Fig. 1(a), and $t_{i}$ are the hopping integrals between an atom on sublattice $A$ and its neighbors. Because both the magnetic field and the curvature are uniform along the whole nanotube, the $t_{i}$ do not depend on the position of the initial $A$ atom. This Hamiltonian can be further expanded around the $K(\tau=1)$ and $K^{\prime}(\tau=$ -1) points [see Fig. 1(b)], yielding

$$
\hat{H}_{\tau}(\kappa)=\hbar v_{F}\left(\begin{array}{cc}
0 & e^{i \tau \theta}\left(\tau \kappa_{\perp}^{\prime}+i \kappa_{\|}^{\prime}\right) \\
e^{-i \tau \theta}\left(\tau \kappa_{\perp}^{\prime}-i \kappa_{\|}^{\prime}\right) & 0
\end{array}\right)
$$

where $\theta$ is the CNT chiral angle, indices $(\perp, \|)$ denote the components perpendicular and parallel to the nanotube axis, respectively, $\hbar v_{F}=3\left|V_{\pi}\right| a_{c} / 2, \kappa=\mathbf{k}-\tau \mathbf{K}$, and

$$
\begin{gathered}
\kappa_{\perp}^{\prime}=\kappa_{\perp}+\tau \Delta k_{\perp}^{c}+\frac{1}{R} \frac{\phi}{\phi_{0}}, \\
\kappa_{\|}^{\prime}=\kappa_{\|}+\tau \Delta k_{\|}^{c} .
\end{gathered}
$$

The last term in (10a) is the Aharonov-Bohm contribution while $\Delta k_{\perp}^{c}$ and $\Delta k_{\|}^{c}$ are due to the curvature,

$$
\begin{aligned}
\Delta k_{\perp}^{c} & =\frac{a_{c}}{4 R^{2}}\left(1+\frac{3}{8} \frac{V_{\sigma}-V_{\pi}}{V_{\pi}}\right) \cos (3 \theta), \\
\Delta k_{\|}^{c} & =-\frac{a_{c}}{4 R^{2}}\left(1+\frac{5}{8} \frac{V_{\sigma}-V_{\pi}}{V_{\pi}}\right) \sin (3 \theta) .
\end{aligned}
$$

In this derivation we used a small angle approximation, $\sin \left(\varphi_{i}-\varphi_{j}\right) \simeq \varphi_{i}-\varphi_{j}$, which is good already for CNTs with $R \gtrsim 5 \AA$. The energy eigenvalues of the Hamiltonian are

$$
E_{ \pm}= \pm \hbar v_{F} \sqrt{\left(\kappa_{\perp}^{\prime}\right)^{2}+\left(\kappa_{\|}^{\prime}\right)^{2}}, \quad \tilde{E}_{ \pm}:=E_{ \pm} /\left(\hbar v_{F}\right) .
$$

Eigenfunctions of the Hamiltonian. The energy eigenstates are a linear combination of Bloch waves. Since we have expanded the Hamiltonian around the $K$ and $K^{\prime}$ points, the corresponding Bloch waves and the coefficients $\eta_{p}$ acquire the index $\tau$. We shall be using

$$
\Phi_{\tau p}(\mathbf{r}, \kappa)=\left\langle\mathbf{r} \mid \Phi_{p}(\tau \mathbf{K}+\kappa)\right\rangle .
$$

Angular boundary condition. The wave function in the angular direction must be periodic. This imposes

$$
\begin{aligned}
\Phi_{\tau p}\left(\left(2 \pi R, x_{\|}\right), \kappa\right) & \stackrel{!}{=} e^{i 2 \pi n} \Phi_{\tau p}\left(\left(0, x_{\|}\right), \kappa\right), \\
& \Rightarrow\left(\tau K_{\perp}+\kappa_{\perp}\right)=\frac{n}{R},
\end{aligned}
$$

which is the standard quantization condition. ${ }^{16}$

Axial boundary condition. The wave function at the ends of the nanotube must satisfy open boundary conditions. We shall derive them for a zigzag nanotube $\left(\theta=0^{\circ}\right)$, but they are valid for any other chirality except armchair, ${ }^{17}$ provided the nanotube edge is a so-called minimal boundary (there are no atoms with only one neighbor).

The Hamiltonian (9) acting on the wave functions $\left|\Phi_{\tau}(\kappa)\right\rangle$, (7) with (13), gives two equations:

$$
\begin{gathered}
e^{i \tau \theta}\left[\tau \kappa_{\perp}^{\prime}+i \kappa_{\|}^{\prime}\right] \eta_{\tau B}(\kappa)=\tilde{E}_{ \pm} \eta_{\tau A}(\kappa), \\
e^{-i \tau \theta}\left[\tau \kappa_{\perp}^{\prime}-i \kappa_{\|}^{\prime}\right] \eta_{\tau A}(\kappa)=\tilde{E}_{ \pm} \eta_{\tau B}(\kappa) .
\end{gathered}
$$

We choose then, up to a normalization factor, $\eta_{\tau A}(\kappa)=\left[\tau \kappa_{\perp}^{\prime}+\right.$ $\left.i \kappa_{\|}^{\prime}\right]$ and $\eta_{\tau B}(\kappa)= \pm\left|\eta_{\tau A}\right| e^{-i \tau \theta}$. We can see from (10b) and (12) that the energies of states with $\kappa_{\|}$and $-\left(\kappa_{\|}+2 \tau \Delta k_{\|}^{c}\right)$ are the same. The energy eigenstate is therefore a linear combination of both:

$$
\begin{aligned}
\psi_{\tau}\left(\mathbf{r}, E_{ \pm}\right)= & a_{1} \Phi_{\tau}\left(\mathbf{r},\left(\kappa_{\perp}, \kappa_{\|}\right)\right) \\
& +a_{2} \Phi_{\tau}\left(\mathbf{r},\left(\kappa_{\perp},-\left(\kappa_{\|}+2 \tau \Delta k_{\|}^{c}\right)\right)\right) .
\end{aligned}
$$

From the structure of the lattice in Fig. 1(a) we see that when the graphene patch is rolled in order to create a zigzag nanotube, the lower CNT edge is formed entirely by $B$ sublattice atoms while the upper edge only by $A$ sublattice atoms. Therefore the wave function on this patch must vanish at the "missing" $A$ atoms below the lower edge $\left(x_{\|}=0\right)$ and $B$ atoms above the upper edge $\left(x_{\|}=L\right)$. The conditions for the sublattice components of $\psi_{\tau}\left(\mathbf{r}, E_{ \pm}\right)$are

$$
\begin{aligned}
& \psi_{\tau A}\left(\left(x_{\perp}, 0\right), E_{ \pm}\right) \stackrel{!}{=} 0, \\
& \quad \rightarrow a_{1}\left(\tau \kappa_{\perp}^{\prime}+i \kappa_{\|}^{\prime}\right)+a_{2}\left(\tau \kappa_{\perp}^{\prime}-i \kappa_{\|}^{\prime}\right)=0,
\end{aligned}
$$




$$
\begin{aligned}
& \psi_{\tau B}\left(\left(x_{\perp}, L\right), E_{ \pm}\right) \stackrel{!}{=} 0, \\
& \quad \rightarrow a_{1} e^{i \kappa_{\|}^{\prime} L-i \tau \Delta k_{\|}^{c} L}+a_{2} e^{-i \kappa_{\|}^{\prime} L-i \tau \Delta k_{\|}^{c} L}=0 .
\end{aligned}
$$

These equations lead to a constraint on the values of $\kappa_{\|}^{\prime}$,

$$
\frac{\tau \kappa_{\perp}^{\prime}+i \kappa_{\|}^{\prime}}{\tau \kappa_{\perp}^{\prime}-i \kappa_{\|}^{\prime}}=e^{2 i \kappa_{\|}^{\prime} L} .
$$

Thus the allowed values of $\kappa_{\|}$depend on $\kappa_{\perp}^{\prime}$, and in particular on the Aharonov-Bohm flux $\phi$. The quantity $\kappa_{\|}^{\prime}$ can be either real or imaginary. If it is real, the wave function describes an extended state. If $\kappa_{\|}^{\prime}$ is imaginary, then $\kappa_{\|}$must be complex, with its real part equal to the second term in (10b). Equation (17) has then one trivial $\left(\kappa_{\|}^{\prime}=0\right)$ and two nontrivial solutions. The latter describe evanescent waves localized near the ends of the nanotube, ${ }^{11}$ because the factor $\exp \left[i(\tau \mathbf{K}+\kappa) \cdot \mathbf{r}_{p i}\right]$ from (6) acquires a damping real part.

The regions where $\kappa_{\|}^{\prime}$ is real or imaginary are determined by the value of $\tau \kappa_{\perp}^{\prime}$ [see Fig. 1(c)]. The two localized state solutions exist if

$$
\text { for } K: \kappa_{\perp}^{\prime}>1 / L, \text { for } K^{\prime}: \kappa_{\perp}^{\prime}<-1 / L .
$$

The spectrum of the CNT is then determined by the value of the magnetic field, which enters into $\kappa_{\perp}^{\prime}$ via (10a). In order to calculate the energy levels, the allowed values of $\kappa_{\|}$must be found from (17) for each value of $\kappa_{\perp}^{\prime}$ separately, as shown in Fig. 1(d) for an $(18,0)$ zigzag CNT.

The analytical method described above gives a remarkable agreement with the spectra obtained by the numerical diagonalization of the nanotube Hamiltonian (1) [see Figs. 2(a) and 3(a)]. The energy of the decaying states tends to 0 with increasing magnetic flux because for $\phi \rightarrow \infty,\left|i \kappa_{\|}^{\prime}\right| \rightarrow \kappa_{\perp}^{\prime}$ for the $K$ point solutions [see Fig. 1(d)]. The CNT spectrum may contain localized $(E=0)$ states even at $\phi=0$, as can be seen in Fig. 2(a) for an $(18,0)$ CNT. If the neighboring $\left(\kappa_{\perp} \neq 0\right)$ subbands lie on the Dirac cone and the condition (18) is fulfilled, then the lowest $\kappa_{\|}$states in the neighboring subbands are localized, while the remaining ones have energies in a higher range, appropriate for their subband. Whether the other subbands lie on the Dirac cone depends on the chirality and diameter of the CNT.

Spin effects. With spin, the Bloch waves (7) become fourcomponent spinors and both the spin-orbit coupling (SOC) and the Zeeman effect must be considered. They will be treated in detail elsewhere. Here we just note that SOC can be taken into account by yet another shift of $\kappa_{\perp}$, while the Zeeman effect splits the energy:

$$
\begin{gathered}
\kappa_{\perp}^{\prime} \rightarrow \kappa_{\perp}^{\prime}+\sigma \Delta k_{\mathrm{SO}}, \quad E_{ \pm} \rightarrow E_{ \pm}+\sigma \mu_{B} \frac{\phi}{\pi R^{2}}, \\
\Delta k_{\mathrm{SO}}=\frac{2 \delta}{R}\left(1+\frac{3}{8} \frac{V_{\sigma}-V_{\pi}}{V_{\pi}}\right),
\end{gathered}
$$

where $\sigma=+1 /-1$ for spin parallel/antiparallel to the CNT axis, $\mu_{B}$ is the Bohr magneton, and $\delta$ is a parameter defining the SOC strength. In our calculations we take $\delta \sim 2.8 \times 10^{-3}$, as, for example, in Ref. 18. The resulting spectra of $(18,0)$ and $(12,9)$ CNTs are shown in Figs. 2(c) and 3(b).

Localization. Equation (18) defines the localization flux $\phi_{\text {loc }}$, at which the extended solutions morph into localized (a)

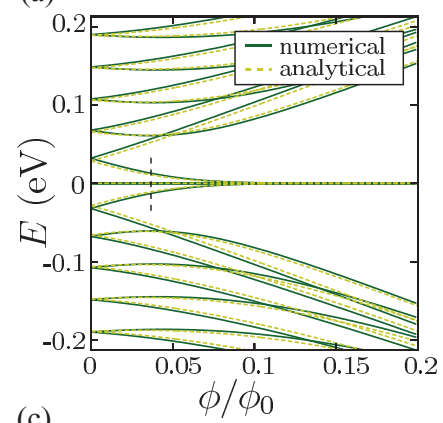

(b)

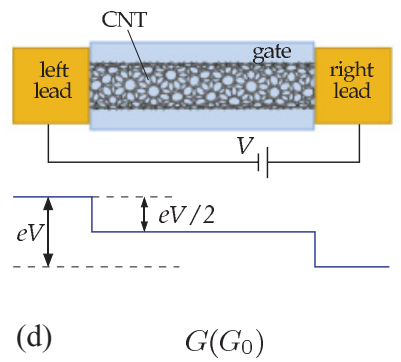

(c)
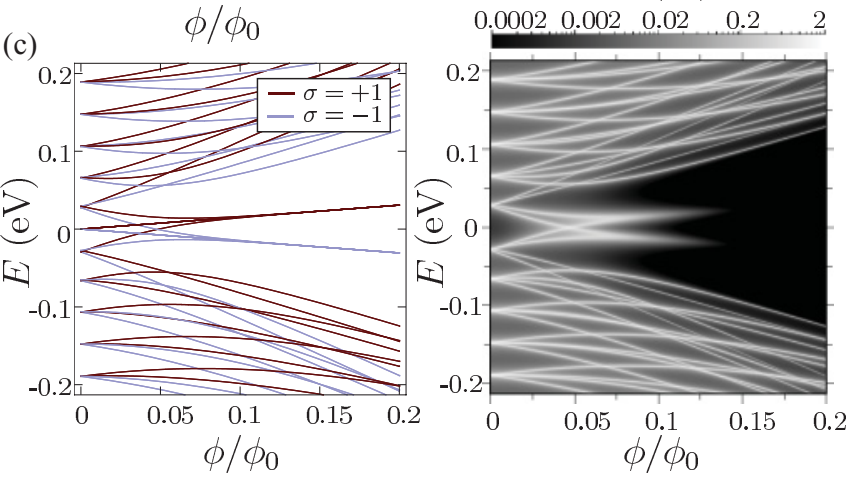

FIG. 2. (Color online) Magnetic-field-induced localization in a $(18,0)$ zigzag CNT with 100 unit cells $\left(L=42.6 \mathrm{~nm}, \theta=0^{\circ}\right)$. (a) The spectra close to the Fermi level obtained by a numerical diagonalization of the real space Hamiltonian (1) and analytically from the Dirac-like dispersion (12) with $\kappa_{\perp}=0$ ( $E \neq 0$ states $)$ and $\kappa_{\perp}= \pm 1 / R$ ( $E \equiv 0$ states), where $\kappa_{\|}$is defined by (17), neglecting the spin. The black dashed line marks the onset of the localization. (b) The setup used for the conductance calculation. (c) Spectra obtained analytically with the electron spin included through (19). (d) Grayscale plot of conductance, in units of conductance quantum $G_{0}=e^{2} / h$, as a function of $\phi$ and the chemical potential $E$, including spin effects.

states. This threshold flux depends on the spin via (19) and using it together with (10a) we obtain

$$
\phi_{\mathrm{loc}}=\tau R\left(\frac{1}{L}-\Delta k_{\perp}^{c}\right) \phi_{0}, \quad \phi_{\mathrm{loc}}^{\sigma}=\phi_{\mathrm{loc}}-\sigma R \Delta k_{\mathrm{SO}} \phi_{0} .
$$

The value of $\phi_{\text {loc }}$ depends on the length of the nanotube. For sufficiently long CNTs the spectra are very close to those of the infinite nanotubes.

The localization induced by the magnetic field is gradual, in principle, allowing the involved states to conduct as long as the two sublattice wave functions overlap. The evolution of an eigenstate in increasing magnetic field is shown in Fig. 3(c), through a sequence of plots of the wave function amplitude at each atom, projected onto $x_{\|}$. The apparent continuity of the curves is due to the overlap between close plot points; near the CNT ends the wave function oscillates with the azimuthal angle $\varphi$ and the individual points can be seen clearly. Initially $\left(\phi=10^{-3} \phi_{0}\right)$ the state is extended; when the magnetic flux reaches $\phi_{\text {loc }}$, its wave function begins to be described by an imaginary solution of (17). With the magnetic field increasing further, the wave function decays exponentially 
(a)
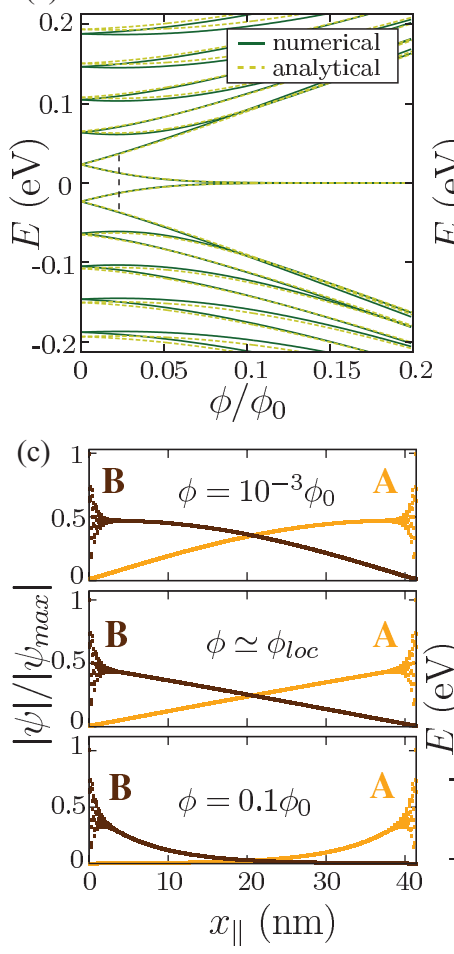

(b)
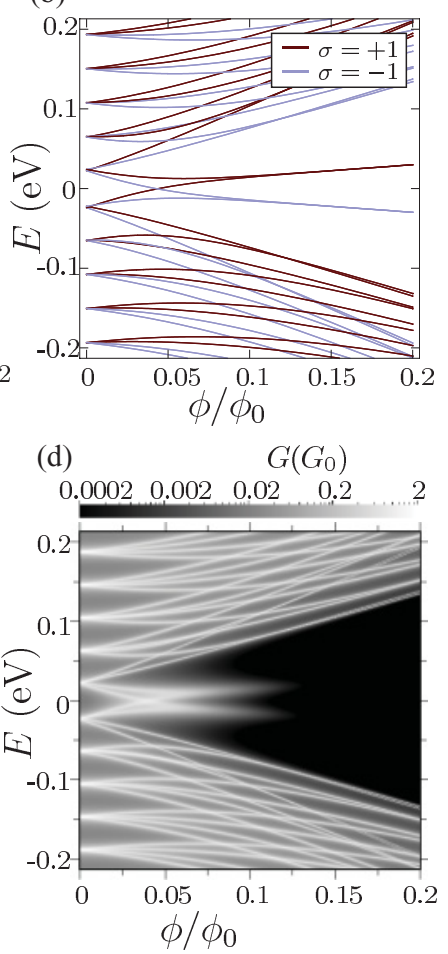

FIG. 3. (Color online) Localization in a $(12,9)$ chiral CNT with 16 unit cells $\left(L=41.5 \mathrm{~nm}, \theta=25^{\circ}\right)$. (a) Comparison of numerical and analytical spectra, neglecting the spin. In this case there are no localized states at $\phi=0$. The dashed line marks the onset of the localization. (b) Analytical spectrum including spin effects. (c) The amplitude of the highest valence eigenstate (obtained numerically) at each atom, projected onto $x_{\|}$, for different values of $\phi$ and neglecting the spin. (d) Conductance as a function of magnetic flux $\phi$ and chemical potential $E$, including spin effects.

with the distance from the CNT ends, the localization becomes complete, and the state ceases to conduct.
The above analysis is confirmed by conductance calculations, with the CNT in a setup shown in Fig. 2(b). We derive the elastic linear response conductance via the Fisher-Lee formula for the quantum mechanical transmission: $G=\frac{e^{2}}{h} \operatorname{Tr}\left\{\Gamma_{L} \mathcal{G} \Gamma_{R} \mathcal{G}^{\dagger}\right\}$, where $\Gamma_{L / R}=i\left(\Sigma_{L / R}-\Sigma_{L / R}^{\dagger}\right), \Sigma_{L / R}$ is the self-energy of the left or right lead, respectively, and $\mathcal{G}$ is the Green function of the central region dressed by the electrodes. For simulating bulk metal electrodes we consider wide band leads, that is, $\Sigma_{\mathrm{WB}}(E)=-i \operatorname{Im} \Sigma\left(E_{F}\right)$. The results shown in Figs. 2(d) and 3(d) were obtained with $\Sigma_{\mathrm{WB}}=$ $-i 0.22 \mathrm{eV}$. In both we see a gradual drop of the conductance of the highest valence and lowest conduction spin states, as they become localized in the increasing magnetic field. The "native" end states of the $(18,0)$ CNT, localized also at $\phi=0$, can be seen in the spectrum in Fig. 2(c), but do not contribute to the conductance, as we expect. The good matching of analytical spectra of isolated CNTs and the conductance peaks [compare Fig. 2(c) with 2(d) and Fig. 3(b) with 3(d)] implies that even with rather strong coupling the CNT is sufficiently distinct from the leads for the transport to be determined by the spectrum of an isolated nanotube.

The magnetic field $B_{\text {loc }}$ corresponding to $\phi_{\text {loc }}$ depends on the nanotube length and radius. For our choice of $V_{\pi}$ and $V_{\sigma}, B_{\text {loc }}$ of CNTs with $R=7 \AA$ and $L=40 \mathrm{~nm}$ ranges from $50 \mathrm{~T}\left(\theta \approx 30^{\circ}\right)$ to $85 \mathrm{~T}\left(\theta=0^{\circ}\right)$. However, for the same nanotubes with $L=500 \mathrm{~nm}$ the value of $B_{\text {loc }}$ drops to 4-42 T. Hence the localization induced by the magnetic field might be detected in currently accessible transport experiments or by spin-resolved STM spectroscopy revealing localized spin states at the nanotube ends. Moreover, the localization induced by a magnetic flux appears to be a chiralityindependent phenomenon, to which only armchair CNTs are immune.

The authors acknowledge the support of the Deutsche Forschungsgemeinschaft under GRK Grant No. 1570.
${ }^{1}$ K. Nakada, M. Fujita, G. Dresselhaus, and M. S. Dresselhaus, Phys. Rev. B 54, 17954 (1996).

${ }^{2}$ L. Brey and H. A. Fertig, Phys. Rev. B 73, 235411 (2006).

${ }^{3}$ K. A. Ritter and J. W. Lyding, Nat. Mater. 8, 235 (2009).

${ }^{4}$ P. Kim, T. W. Odom, J.-L. Huang, and C. M. Lieber, Phys. Rev. Lett. 82, 1225 (1999).

${ }^{5}$ S. Ryu and Y. Hatsugai, Phys. Rev. B 67, 165410 (2003).

${ }^{6}$ K. Sasaki, K. Sato, R. Saito, J. Jiang, S. Onari, and Y. Tanaka, Phys. Rev. B 75, 235430 (2007).

${ }^{7}$ A. Mañanes, F. Duque, A. Ayuela, M. J. López, and J. A. Alonso, Phys. Rev. B 78, 035432 (2008).

${ }^{8}$ J. Wu and F. Hagelberg, Phys. Rev. B 79, 115436 (2009).

${ }^{9}$ L. Rosales, M. Pacheco, Z. Barticevic, C. G. Rocha, and A. Latgé, Phys. Rev. B 75, 165401 (2007).

${ }^{10}$ H. Santos, A. Ayuela, W. Jaskólski, M. Pelc, and L. Chico, Phys. Rev. B 80, 035436 (2009).
${ }^{11}$ K. Sasaki, S. Murakami, R. Saito, and Y. Kawazoe, Phys. Rev. B 71, 195401 (2005).

${ }^{12}$ K. Sasaki, M. Suzuki, and R. Saito, Phys. Rev. B 77, 045138 (2008).

${ }^{13}$ T. Ando, J. Phys. Soc. Jpn 69, 1757 (2000).

${ }^{14}$ S. Koller, L. Mayrhofer, and M. Grifoni, New J. Phys. 12, 033038 (2010).

${ }^{15}$ D. V. Bulaev, B. Trauzettel, and D. Loss, Phys. Rev. B 77, 235301 (2008).

${ }^{16}$ R. Saito, G. Dresselhaus, and M. S. Dresselhaus, Physical Properties of Carbon Nanotubes (Imperial College Press, London, 1998).

${ }^{17}$ A. R. Akhmerov and C. W. J. Beenakker, Phys. Rev. B 77, 085423 (2008).

${ }^{18}$ S. H. Jhang, M. Marganska, Y. Skourski, D. Preusche, B. Witkamp, M. Grifoni, H. van der Zant, J. Wosnitza, and C. Strunk, Phys. Rev. B 82, 041404 (2010). 